\title{
Trifrequency Reconfigurable Linear Irregular Array with Beam Deflection Capability in $\mathrm{X} / \mathrm{Ku} / \mathrm{Ka}-\mathrm{Bands}$
}

\author{
Ming Li $\mathbb{D}^{1},{ }^{1}$ Haiping Wei, ${ }^{2}$ Jiahao Zhao, ${ }^{1}$ and Qingchang Tao ${ }^{1}{ }^{1}$ \\ ${ }^{1}$ State Key Laboratory of Precision Measurement Technology and Instruments, Department of Precision Instrument, \\ Tsinghua University, Beijing 100084, China \\ ${ }^{2}$ National Key Laboratory of Science and Technology on Aerospace Intelligent Control, \\ Beijing Aerospace Automatic Control Institute, Beijing 100854, China \\ Correspondence should be addressed to Qingchang Tao; taoqingchang@mail.tsinghua.edu.cn
}

Received 2 April 2020; Revised 31 May 2020; Accepted 8 June 2020; Published 24 June 2020

Academic Editor: Renato Cicchetti

Copyright (C 2020 Ming Li et al. This is an open access article distributed under the Creative Commons Attribution License, which permits unrestricted use, distribution, and reproduction in any medium, provided the original work is properly cited.

\begin{abstract}
This paper proposes a trifrequency reconfigurable antenna (FRA), which can work in the X-band, Ku-band, and Ka-band, by controlling only two RF MEMS switches. The antenna element has a frequency ratio beyond $3: 1$ and provides a good candidate for the frequency reconfigurable antenna array, since the size of the antenna is reduced by loading multiple metal shorting holes between the antenna radiating surface and the ground plate, and the overall size is only $0.14 \lambda_{\mathrm{X}} \times 0.35 \lambda_{\mathrm{X}}\left(\lambda_{\mathrm{X}}\right.$ is the free-space wavelength at $8.6 \mathrm{GHz}$ ). Based on the proposed FRA element, a $1 \times 16$ linear irregular frequency reconfigurable antenna array (FRAA) with beam deflection ability is designed, which effectively addresses the element spacing problem in the optimization of the array. In addition, the close-coupling in X-band and the grating lobe caused by the long distance of array element spacing in Ka-band are comprehensively considered. With uniform amplitude feeding network, the sidelobe level is below $-15 \mathrm{~dB}$ under beam deflection. Moreover, both FRA elements and FRAA prototypes have been fabricated and measured to verify their superiority. Good agreements are obtained between simulated and measured results, which indicates that the antenna has potential application in the future multifrequency wireless communication and intelligent radar anti-interference fields.
\end{abstract}

\section{Introduction}

IWith the rapid development of wireless communication and intelligent radar technology, antennas have been desired to be multifunctional, intelligent, miniaturized, and low cost. However, traditional single-function antenna is difficult to satisfy these new requirements. Increasing the number of single-function antennas to implement multifunctional RFintegrated equipment can inevitably lead to many problems; that is, the increase in the area occupied by the antenna makes it difficult to achieve miniaturization and the stealth performance of the aircraft. Moreover, the increase in equipment weight and cost becomes the main obstacle in the green application and development of the multifunctional antenna. In such case, reconfigurable antenna, by mechanically or electrically changing its architecture, can effectively change its performance characteristics (resonant frequency, radiation pattern, polarization, etc.) [1] and therefore provides a promising candidate to realize intelligent RF front end.

Frequency reconfigurable antenna (FRA) has the advantages of high spectrum utilization and strong anti-interference ability in the frequency domain. The resonant frequency can be adjusted according to the working environment. Previously, FRA has received widespread attention from the industrial sector and academia. The characteristics of FRA have been verified and FRAs implemented in various forms, including dipole [2], slot [3, 4], Vivaldi [5], patch $[6,7]$, fractal [8], pixels [9-11], and reflection array [12], with their applications in radar [13], wireless communication $[4,9]$, cognitive radio [2, 5], aircraft conformal antenna [11], and satellite communication and navigation $[7,12]$.

From the previous research mentioned previously, we know that the previously proposed FRA element mostly 
focused on the low-frequency band, and the frequency ratio was generally less than $3: 1$ [2-11]. However, relatively few reconfigurable antennas for $\mathrm{Ku}$ or higher frequency bands and frequencies greater than $3: 1$ have been put forward [14]. In response to the requirements of wireless communications, remote sensing, and radar for frequency reconfigurable antenna arrays, a number of researches have also been carried out [14-19]. However, the half-wavelength constraint condition of the spacing between the traditional phased array antenna elements poses great challenges to the layout of the frequency reconstructed antenna array. Arraying in a low-frequency state makes the high-frequency spacing too large, but in a high-frequency state it can cause coupling in the low frequency $[16,19]$. Therefore, trifrequency FRAA with beam deflection capability covering Kaband and frequency ratio greater than $3: 1$ is urgently desired.

In this paper, a novel trifrequency $\mathrm{X} / \mathrm{Ku} / \mathrm{Ka}$ FRA element is proposed, and a $1 \times 16$ linear irregular FRAA is designed based on this element. The proposed design has the following advantages: (1) The FRA unit uses two RF MEMS switches to achieve frequency switching in $\mathrm{X}$-band, $\mathrm{Ku}$ band, and Ka-band, and a frequency ratio of 3.57:1 is achieved. (2) The loading of multiple shorting metal holes between the radiation patch and the ground plate reduces the overall size of the FRA to $0.14 \lambda_{\mathrm{X}} \times 0.35 \lambda_{\mathrm{X}}\left(\lambda_{\mathrm{X}}\right.$ is the freespace wavelength at $8.6 \mathrm{GHz}$ ), at the lowest frequency. Thus, it provides a good candidate for the optimization of the element spacing of the FRAA. (3) Improved fruit-fly optimization algorithm (FOA) together with MATLAB-HFSS cosimulation is used to optimize the element spacing of a $1 \times 16$ linear irregular FRAA with beam deflection ability in the same direction in X-band, Ku-band, and Ka-band. The tough spacing problem in the optimization of the traditional FRAA is effectively addressed by the proposed method in this paper, and the sidelobe levels of different frequency reconfigurability states are all lower than $-15 \mathrm{~dB}$.

This paper is organized as follows. In Section 2, the design of the FRA element is introduced, and its frequency reconfigurable performance is simulated and analysed. In Section 3, the design and optimization of linear irregular frequency reconfigurable arrays are discussed in detail, and the sidelobe levels of equally spaced antenna arrays are compared and analysed. The production and testing of FRA and FRAA prototypes are introduced in Section 4. Finally, conclusion is drawn in Section 5.

\section{Design of FRA Element}

2.1. Basic Structure Design. It is well known that the operating frequency of the antenna depends on the equivalent electrical length of the radiator. Generally speaking, the longer the equivalent electrical length, the lower the operating frequency. Motivated by that, an easy way to achieve multiband reconfigurable antenna can start from the smallest size corresponding to the highest frequency, that is, by connecting additional patches with switches and by adjusting the overall size of the antenna by controlling the switches' On and Off state. According to this principle, the basic structure of the proposed FRA element is shown in Figure 1. Three multiscale microstrip patches are printed on the Rogers 4350B dielectric substrate, whose dielectric constant is 3.66 , loss tangent is 0.004 , and thickness is $0.508 \mathrm{~mm}$. It is fed by using a coaxial line. When designing the basic structure of the FRA element, the two red rectangles of $2.0 \mathrm{~mm} \times 1.1 \mathrm{~mm}$ in Figure 1 indicate that $\mathrm{RF}$ switches are replaced by copper plates to adjust the antenna topology. The connection or disconnection of the metal strip is equivalent to the On/Off function of the switch. When S1 is On and S2 is Off, the FRA element can work in X-band. When S1 is Off and S2 is On, the FRA element can work in Ku-band. Otherwise, when both S1 and S2 switches are Off, the FRA element will work in Ka-band. Figure 2 shows the optimized process of the size of the three radiators.

In addition, the antenna is utilized to construct a linear array working at the Ka-band (highest), which limits the size of the antenna element in the array dimension. The recent research shows that miniaturization of microstrip antennas can be achieved by shorting surfaces, shorting strips, or shorting probes $[6,20]$. Inspired by that, five metal shorting holes between the microstrip patch and the ground plate are employed to reduce the overall antenna size in this paper, and through the optimization of the diameter and spacing of the metal shorting holes, a significant reduction in bandwidth is avoided, the optimized results of which are shown in Figure 2(c).

Figure 3 shows the simulation results of reflection coefficients at different frequency reconstruction states. It can be seen that FRA can work in X-band, Ku-band, and Kaband. Taking the reflection coefficient less than $-10 \mathrm{~dB}$ as the reference, the impedance bandwidth and center frequency are $106 \mathrm{MHz}$ (8.6 GHz), $230 \mathrm{MHz}(17.1 \mathrm{GHz})$, and $1900 \mathrm{MHz}$ $(31.1 \mathrm{GHz})$, respectively. Moreover, the reconstruction frequency ratio of the FRA reaches $3.61: 1$, and the size is $5.0 \mathrm{~mm} \times 12.3 \mathrm{~mm}$, which is only $0.143 \lambda_{\mathrm{X}} \times 0.353 \lambda_{\mathrm{X}}$, at the lowest frequency $(8.6 \mathrm{GHz})$.

2.2. FRA Integrated RF MEMS Switch Design. The coplanar waveguide capacitive MEMS switch proposed by the author's research group is used. The substrate material is highresistance silicon. The insulating layer is formed by thermal oxidation of a silicon substrate, whose main material component is silicon oxide. A detailed description of the switch can be found in [21].

The structure of the FRA integrated RF MEMS switch is shown in Figure 4. Based on the antenna structure in the ideal switching state, a prepreg layer for lamination is added under the ground plate, and a Rogers 4350B dielectric substrate with a thickness of $0.508 \mathrm{~mm}$ is introduced, which is used to route the coaxial feed port and the DC bias lines of the RF MEMS switches. Gold wire with 20 um diameter is used to bond RF MEMS switches and microstrip patches.

The transition structure of the RF MEMS switch integrated into the microstrip antenna is shown in Figure 5, and the transition structures of the switches S1 and S2 have different sizes as shown in Table 1. The CPW center signal line of the RF MEMS switch is connected to the microstrip 


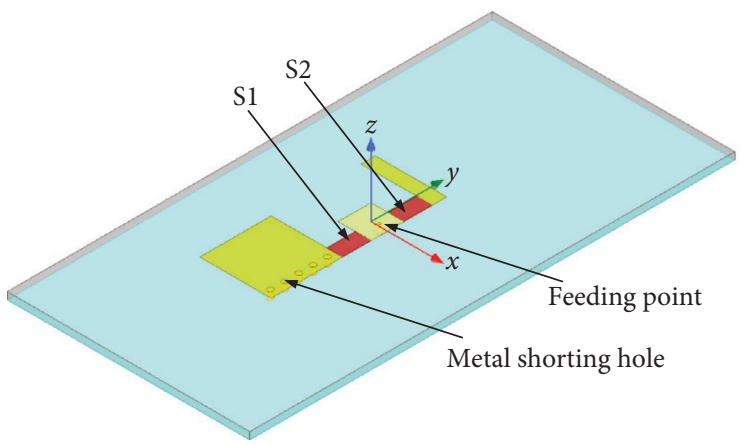

(a)

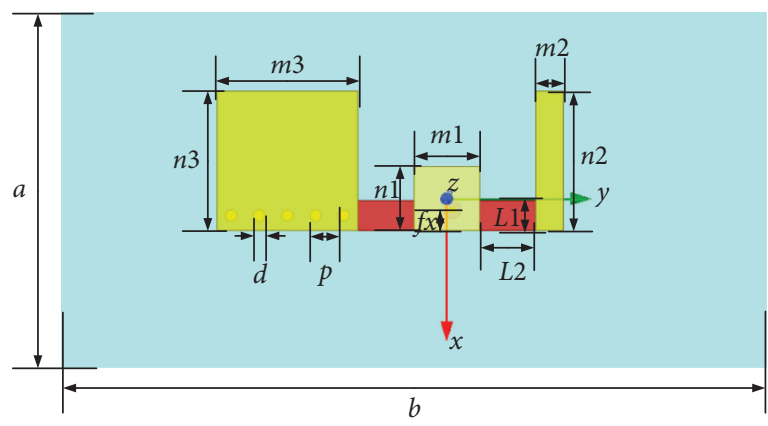

(b)

Figure 1: Geometry of the proposed FRA element. (a) 3D view. (b) Front view (unit (mm): $a=17.0, b=34.0, m 1=n 1=2.3, m 2=1.0$, $n 2=5.0, m 3=n 3=5.0, d=0.4, p=1.0, L 1=1.1$, and $L 2=2.0)$.

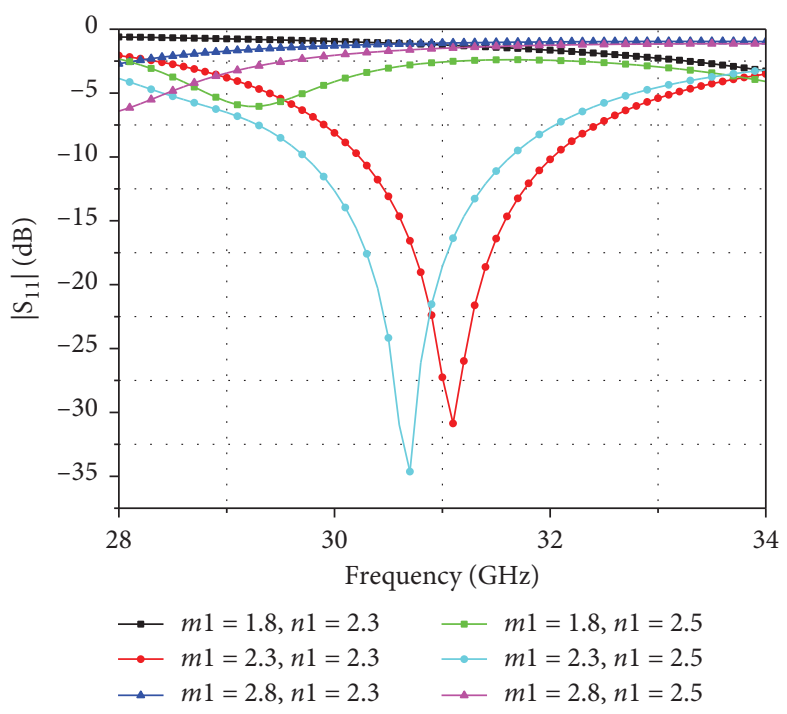

(a)

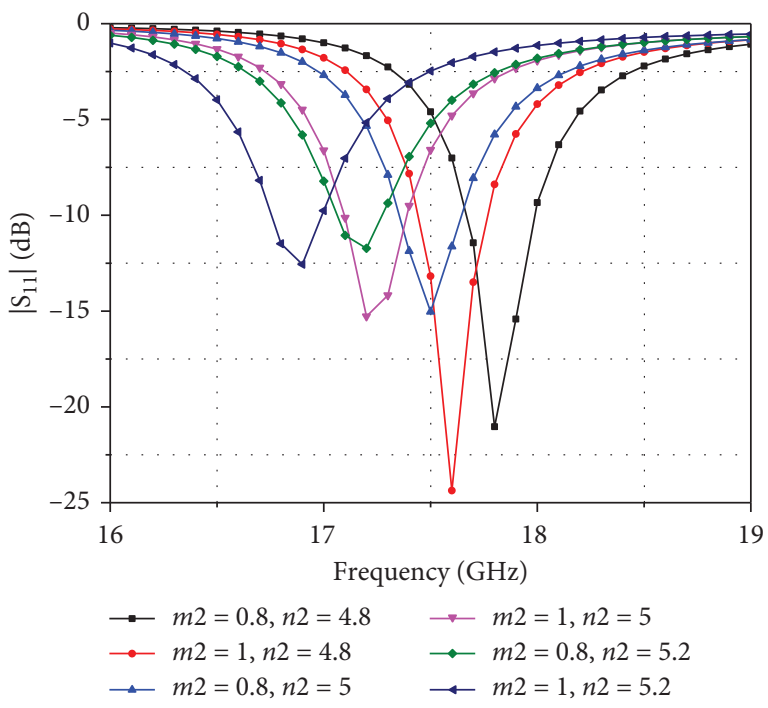

(b)

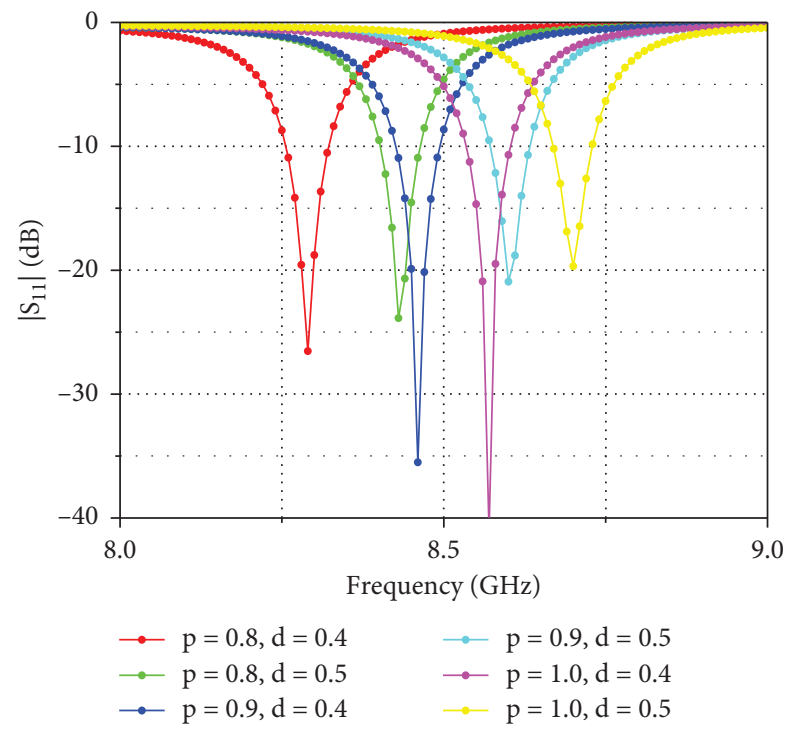

(c)

FIGURE 2: Optimized results of the three radiators. (a) Ka-band. (b) Ku-band. (c) X-band. 


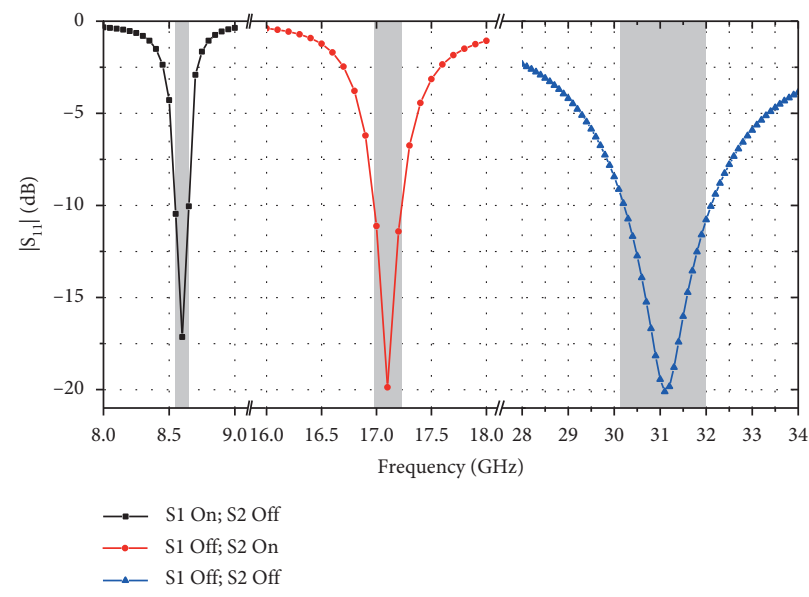

FIGURE 3: Simulation results of reflection coefficients at different frequency reconstruction states.

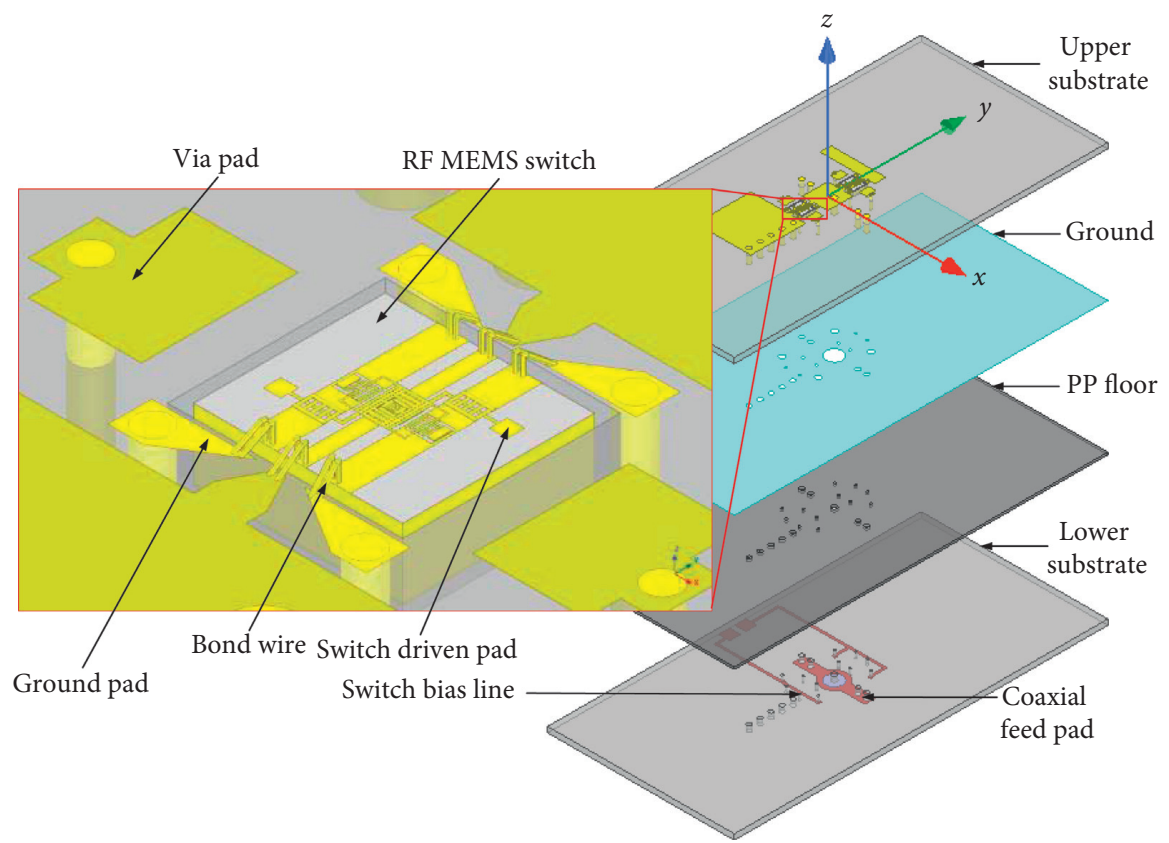

FIGURE 4: Structure of the FRA integrated RF MEMS switches.

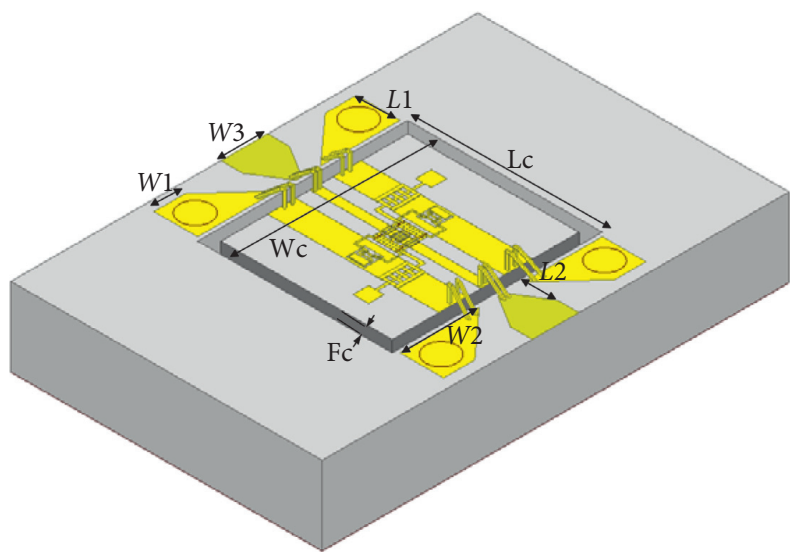

FIGURE 5: Transition structure of RF MEMS switch integrated in microstrip antenna.
TABLE 1: Parameters of the transition structure of RF MEMS switch integrated in microstrip antenna.

\begin{tabular}{lcc}
\hline Parameters & S1 $(\mathrm{mm})$ & S2 $(\mathrm{mm})$ \\
\hline L1 & 0.12 & 0.14 \\
L2 & 0.25 & 0.28 \\
$W 1$ & 0.20 & 0.20 \\
$W 2$ & 0.60 & 0.51 \\
$W 3$ & 0.30 & 0.30 \\
\hline
\end{tabular}

antenna radiation patch through a piece of trapezoidal gradient impedance transformation structure to achieve impedance matching. In addition, a rectangular slot for switch installation is designed with a gap of $\mathrm{Fc}=75 \mu \mathrm{m}$, an

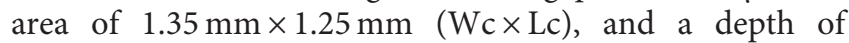
$410 \mu \mathrm{m}$. 
Simulation results of the reflection coefficient of FRA integrated RF MEMS switches are shown in Figure 6. Under different frequency reconstruction states, the impedance bandwidth and center frequency of the FRA are $110 \mathrm{MHz}$ $(8.7 \mathrm{GHz}), 226 \mathrm{MHz}(17.0 \mathrm{GHz})$, and $1902 \mathrm{MHz}(31.1 \mathrm{GHz})$, respectively. The reflection coefficients of the antenna using the ideal switch and the antenna using the RF MEMS switch are evaluated and compared, and the basically matched frequency reconstruction characteristics are obtained, which also indicates that the effective integration of the switches is achieved through introducing the switch transition structure.

\section{Design of FRA Array}

3.1. Spacing Optimization of FRA Element. As shown in Figure 7 , consider an $N$-element linear scan array distributed along the $x$-axis [22], let $d$ be the distance between the array elements, and let $\theta_{B}$ be the scan angle; then the total field strength at the observation point $P$ in the far field of the array can be expressed as

$$
E_{n}=f_{e}(\theta) \sum_{n=1}^{N} a_{n} e^{j(2 \pi / \lambda)(n-1) d\left(\sin \theta-\sin \theta_{B}\right)},
$$

where $f_{e}(\theta)$ is the radiation pattern of a single antenna element, $\theta$ is the angle from boresight, $\lambda$ is the wavelength, and $a_{n}$ represents the amplitude weighting coefficient of each array element.

If the array is required not to generate grating lobes, $d$ has to satisfy the following conditions:

$$
d<\frac{\lambda}{1+|\sin \theta B|} \text {. }
$$

It can be known from (2) that if the frequency reconfigurable linear array is designed with the array element spacing in the Ka-band, the constraint conditions for the element interval without the grating lobe are satisfied in both $\mathrm{X}$-band and $\mathrm{Ku}$-band. However, for the X-band reconstruction state, since the antenna array units are too dense, an increase in coupling effects and a decrease in radiation efficiency and gain can be resulted. If the array spacing design is performed in the X-band, the sidelobe level of the Ka-band will inevitably be reduced, and even grating lobes can appear. Therefore, the optimization of the array element spacing becomes a key factor in the design of trifrequency FRAA.

Genetic algorithm and full wave analysis software HFSS were jointly used to solve the optimization problem of reconfigurable antenna [23]. An improved fruit-fly optimization algorithm (FOA) is proposed to reduce the sidelobe level for antenna array synthesis in [24]. In this paper, the distances between FRA elements are taken as the optimization objectives, and the improved fruit-fly optimization algorithm and MATLAB-HFSS joint simulation are used to optimize the line irregular FRAA. The spacing optimization flowchart of the linear irregular FRAA elements is shown in Figure 8.

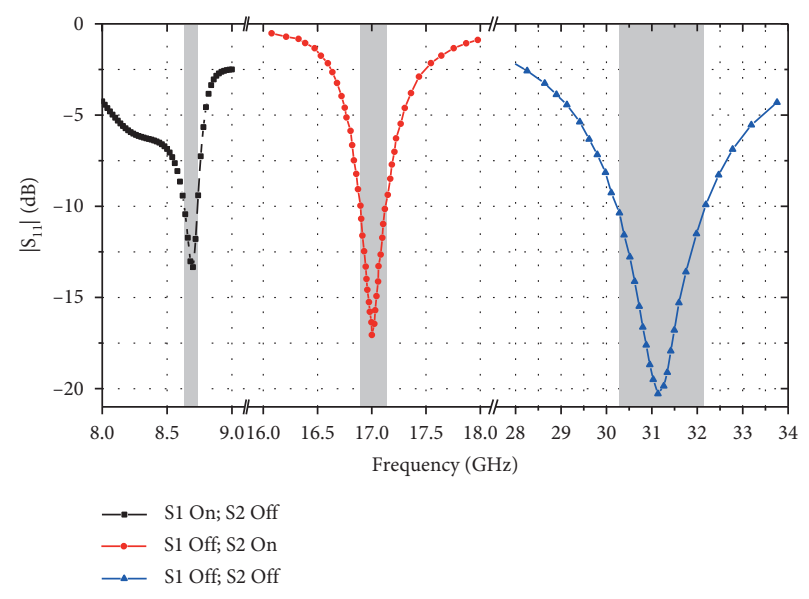

FIGURE 6: Simulation results of the reflection coefficient of FRA integrated RF MEMS switches.

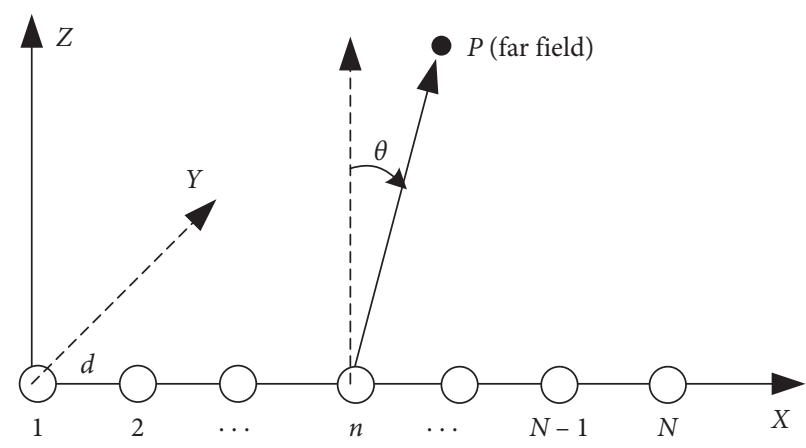

FIgURE 7: Schematic diagram of linear array antenna.

3.2. FRAA Design. Based on the FRA element designed previously and the array unit spacing optimization process shown in Figure 9, a $1 \times 16$ linear irregular FRAA with beam deflection capability is designed. Then, the optimization objective is denoted as $D=\left[d_{1}, d_{2}, \ldots d_{i}, \ldots, d_{15}\right]$, where $d_{i}$ is the spacing between the $i$-th and the $(i+1)$-th FRA element. The optimization interval of the element spacing is $6.5 \mathrm{~mm}$ to $20 \mathrm{~mm}$. The optimization objective function of this FRAA is defined as follows:

$$
\begin{aligned}
& \min _{D}\left|\operatorname{maxSLL}_{\mathrm{X}, \mathrm{Ku}, \mathrm{Ka}}(D)-\mathrm{SLL}_{\mathrm{dgn}}\right|, \\
& \text { s.t. } \quad D=\left[d_{1}, d_{2}, \ldots d_{i}, \ldots, d_{15}\right], \quad d_{i} \in(6.5 \mathrm{~mm}, 20 \mathrm{~mm}),
\end{aligned}
$$

where $\operatorname{maxSLL}_{\mathrm{X}, \mathrm{Ku}, \mathrm{Ka}}(D)$ is the maximum sidelobe level of the array pattern at the three frequency bands and $\mathrm{SLL}_{\mathrm{dgn}}$ is the optimization constraint for the sidelobe level, which is $-15 \mathrm{~dB}$ in the optimization.

In order to simplify the design optimization process of the $1 \times 16$ linear irregular array, copper microstrip lines are used instead of RF MEMS switches. The radiating surface structure is shown in Figure 9. The 16 red rectangles and 16 blue rectangles, respectively, represent a switch group $\mathrm{K} 1$ and a switch group K2 composed of copper strips. The optimized element spacing and the required phases are provided in Figure 10. The length of the effective radiation aperture of the FRAA is $128.0 \mathrm{~mm}$. 


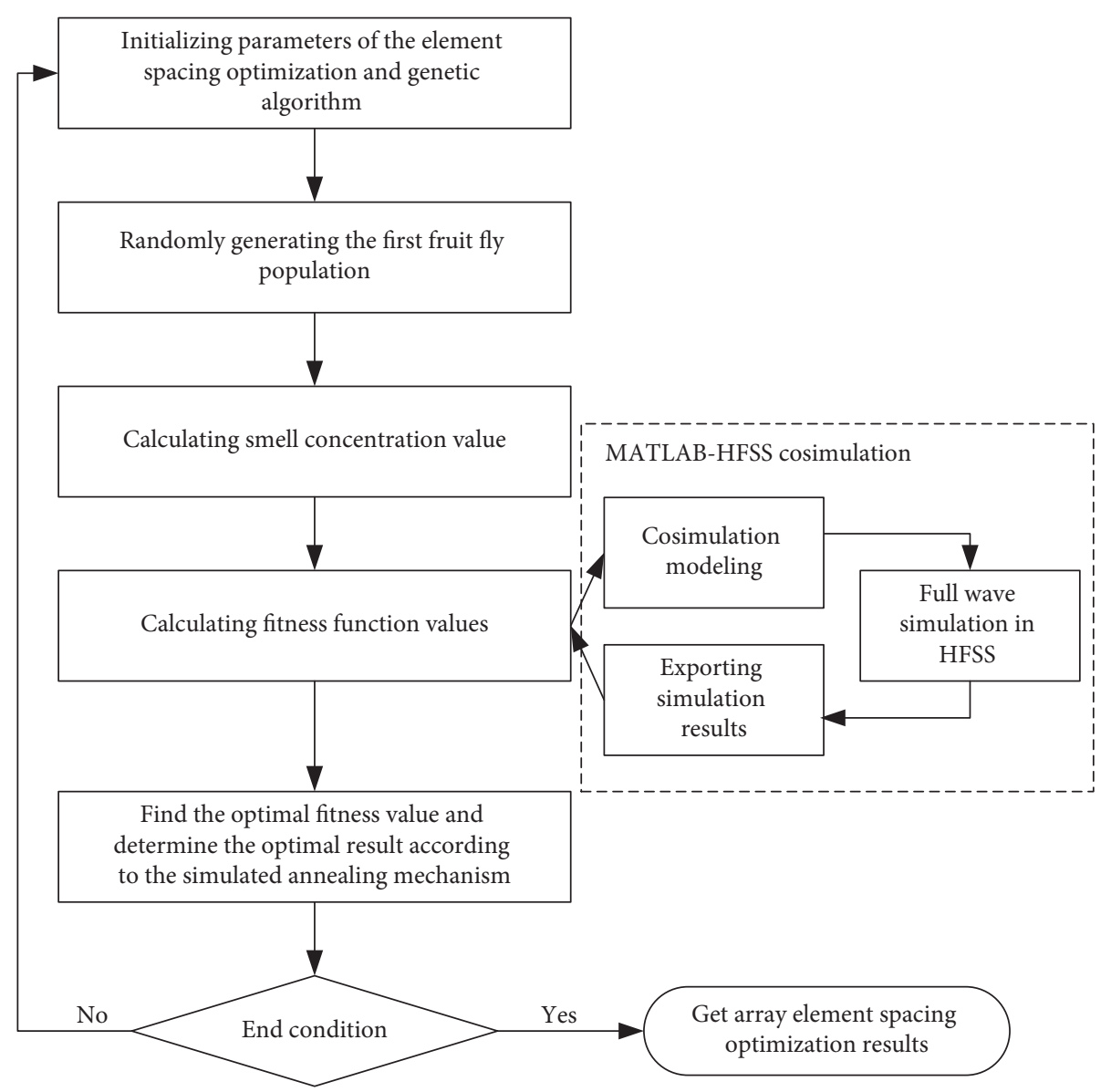

FIGURE 8: Flowchart of spacing optimization of the linear irregular FRAA elements.

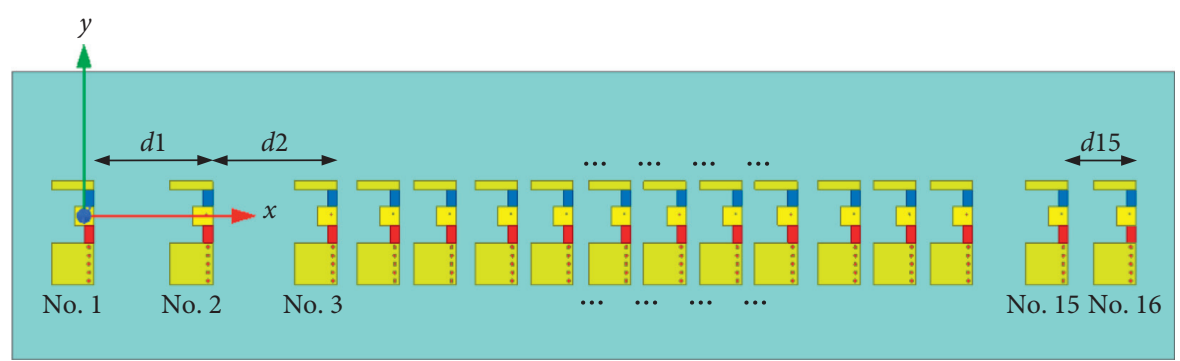

FIGURE 9: The schematic of the $1 \times 16$ linear irregular FRAA.

When $\mathrm{K} 1$ is $\mathrm{On}$ and $\mathrm{K} 2$ is Off, the FRAA works in $\mathrm{X}$-band. When $\mathrm{K} 1$ is Off and $\mathrm{K} 2$ is On, the FRAA can work in $\mathrm{Ku}$-band. In addition, when both $\mathrm{K} 1$ and $\mathrm{K} 2$ are Off, the FRAA will work in Ka-band. From the results of the reflection coefficients of different frequency reconstruction states in Figure 11, it can be seen that the impedance bandwidth and center frequency of the FRAA are $110.0 \mathrm{MHz}$ $(8.7 \mathrm{GHz}), \quad 195.0 \mathrm{MHz} \quad(17.1 \mathrm{GHz}), \quad$ and $\quad 1800 \mathrm{MHz}$ $(30.0 \mathrm{GHz})$, respectively. Moreover, the reconstruction frequency ratio of the FRA reaches $3.45: 1$. The frequency reconstruction capability of the antenna array and the frequency reconfigurable antenna unit is basically the same.

Simulation results of the radiation pattern of the FRAA under constant amplitude feeding conditions are shown in
Figure 12. Corresponding to the reconstruction states of Kaband, Ku-band, and X-band, the main polarization states are all vertical polarization, and the cross-polarization levels are $-15.8 \mathrm{~dB},-23.3 \mathrm{~dB}$, and $-17.5 \mathrm{~dB}$, respectively. Besides, the sidelobe levels are $-15.7 \mathrm{~dB},-17.0 \mathrm{~dB}$, and $-16.2 \mathrm{~dB}$, respectively. The FRAA gains are $18.1 \mathrm{~dB}, 17.0 \mathrm{~dB}$, and $12.5 \mathrm{~dB}$. In addition, the pattern beams deflection of the three states is the same; that is, $\theta_{x o z}=20^{\circ}$.

For fair comparison, a conventional uniform-pitch phased array is designed under the condition that the effective radiation aperture of the antenna array is $128 \mathrm{~mm}$ with uniform amplitude excitation. The radiating surface structure of the uniform FRAA is shown in Figure 13. The states of the switch groups corresponding to different 


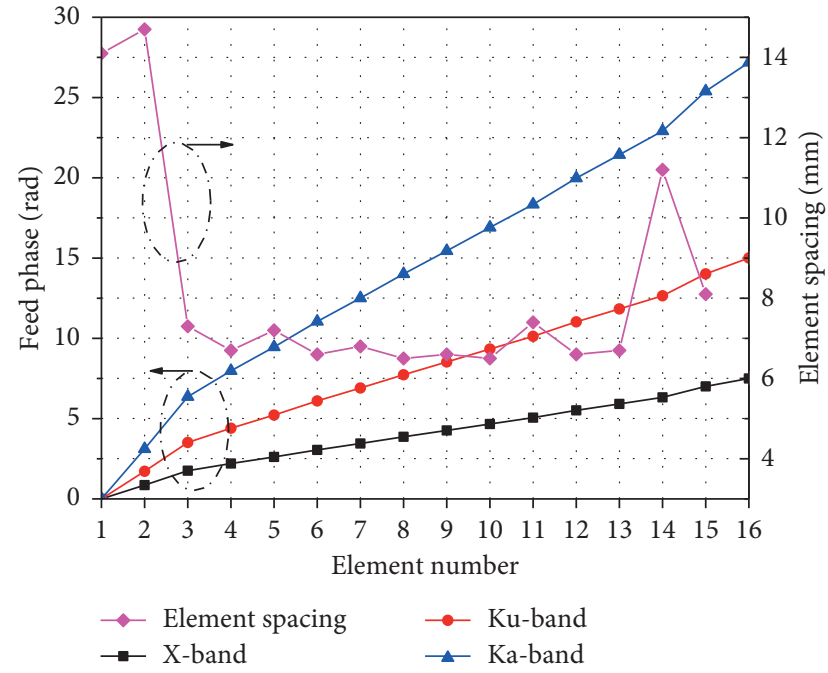

FIGURE 10: Optimization results of FRAA element spacing and port feed phases.

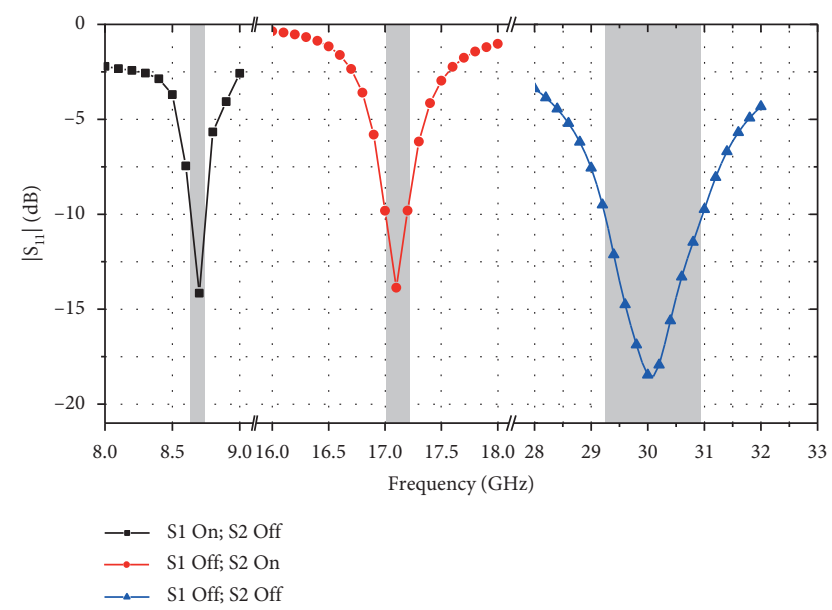

FIGURE 11: Simulation results of the reflection coefficient of FRAA.

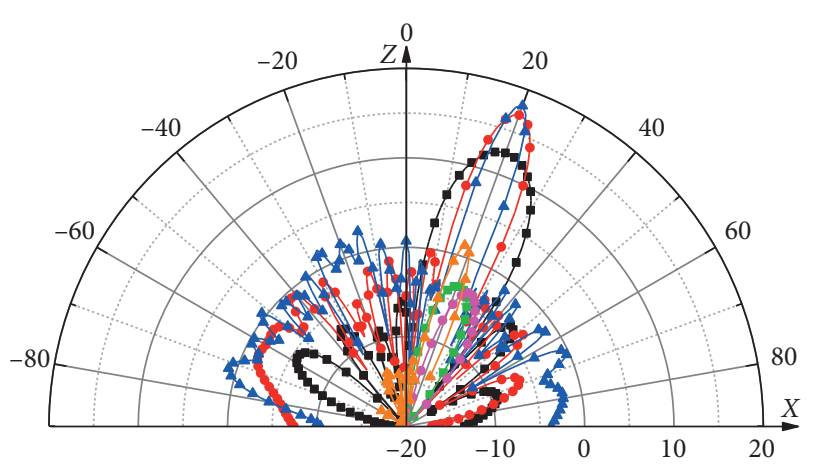

(dB)

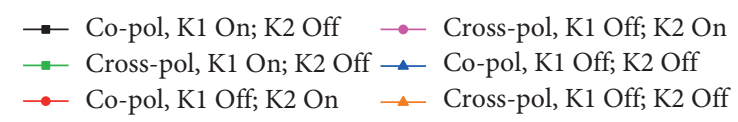

FIGURE 12: Simulated radiation pattern of the linear irregular FRAA with the beam steering angles of $\theta_{x o z}=20^{\circ}$. frequency reconstructions are the same as those of the irregular array shown in Figure 9. However, all the spacing between the array elements is $8.2 \mathrm{~mm}$.

Figure 14 shows the radiation pattern of the uniform array. Due to the effect of the array factor, the beam deflection is still $\theta_{x o z}=20^{\circ}$. Moreover, since the array aperture and the number of array elements are not changed, its antenna gains and $3 \mathrm{~dB}$ beam width are almost the same as those of the irregular FRAA. However, it can also be seen that the sidelobe levels are $-12.7 \mathrm{~dB},-12.5 \mathrm{~dB}$, and $-12.3 \mathrm{~dB}$ in Ka-band, Ku-band, and X-band, respectively. The increase in the sidelobe levels of the uniform array relative to the irregular FRAA is more than $3 \mathrm{~dB}$, as shown in Figure 15. It should be noted that when the uniform FRAA works in the Ka-band state, an obvious grating lobe appears at $-53^{\circ}$ in the pattern, which mainly because the array element spacing exceeds the limit condition of (2). When the array works in the Ka-band and the scanning angle is $20^{\circ}$, the interval between the arrays should be controlled within $7.2 \mathrm{~mm}$ to avoid the generation of grating lobes. However, under this constraint, the array element spacing in the X-band operating state is less than $0.2 \lambda_{X}$, which can definitely result in the strong coupling effect between array elements.

To show the superiority, the irregular FRAA proposed in this paper is compared with other counterparts as listed in Table 2. The tunable FRA element has advantages in the frequency reconstruction range and the number of switches used. In addition, the irregular FRAA optimized by the joint intelligent algorithm and MATLAB-HFSS also achieves a relatively low sidelobe level with uniform amplitude feeding. It can also be seen that the proposed FRA element structure has good potential in forming an array, and the implementation of the FRAA has the capability to solve the problem of the constraint of the element spacing in the current FRAA design.

\section{Fabrication and Measurement}

Based on the design of the FRA element in Section 2, three prototypes with ideal switch of the FRA element processed are shown in Figure 16. The feed port is equipped with a 2.92-KFD0705 RF connector that can support frequencies up to $40 \mathrm{GHz}$, covering the whole frequency reconfigurable range. To test the reflection coefficient of the antenna, Agilent A5244A vector network analyser, with a maximum adaptive frequency of $50 \mathrm{GHz}$, is used. The reflection coefficient test results of the FRA unit are shown in Figure 17. The results of the prototype test and theoretical simulation are in good agreement. The center frequency and impedance bandwidth of different frequency reconstruction states are almost unchanged compared with the simulation results, which proves that the FRA unit proposed in this paper has good frequency reconstruction characteristics.

According to the FRAA design in Section 3, three types of ideal switch FRAA prototypes are processed, and a uniform amplitude feeding network is added to each prototype. By simultaneously optimizing the feed line 


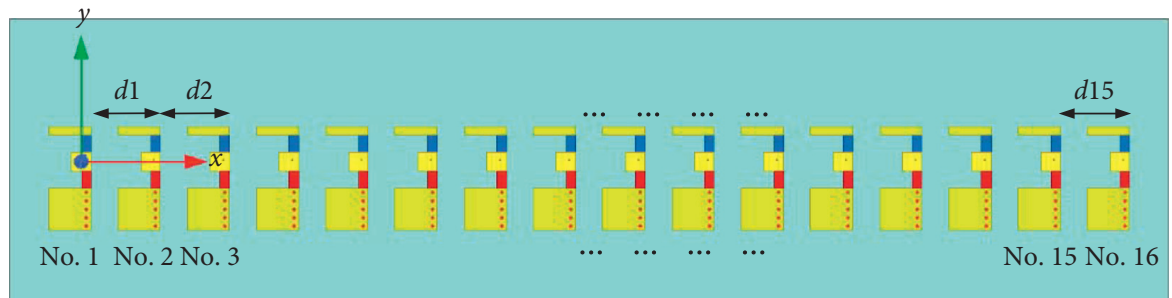

FIgURE 13: The schematic of the $1 \times 16$ linear uniform FRAA.

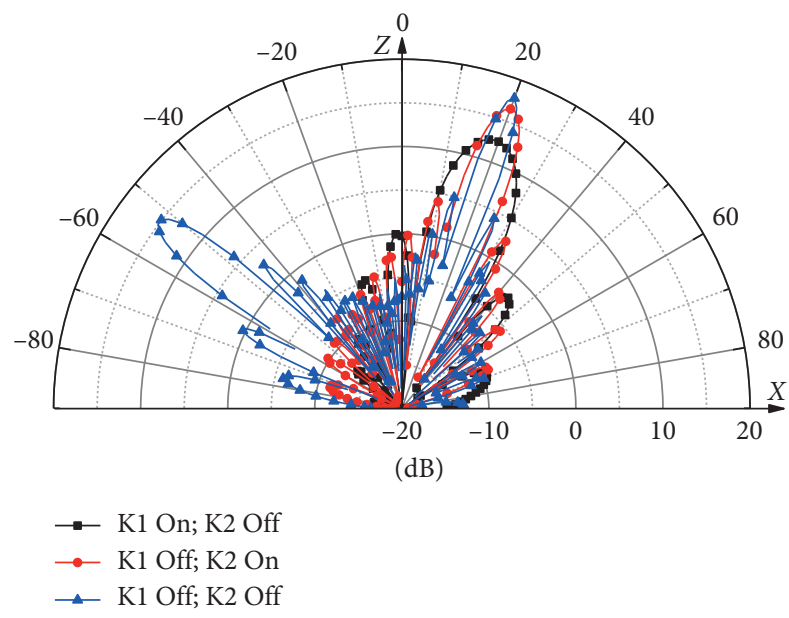

FIgURE 14: Simulated radiation pattern of the uniform FRAA with the beam steering angles of $\theta_{x o z}=20^{\circ}$.

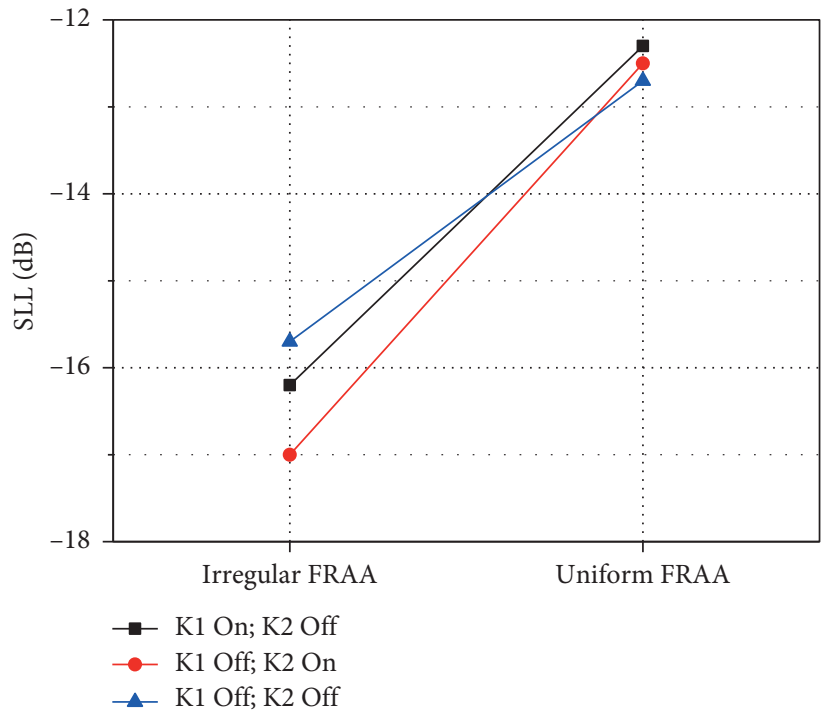

FIgURE 15: Comparison of sidelobe levels in irregular and uniform FRAA.

width of power divider and the length of the delay line, the amplitude consistency of each output port is achieved. Moreover, the feed phase difference of the FRAA prototype for each frequency reconstruction state is determined by Figure 10. The structure of the FRAA prototype is shown in Figure 18, and the processed FRAA prototypes are shown in Figure 19.
The radiation patterns of FRAA prototypes are tested in a microwave shielded room, as shown in Figure 20. Figure 21 shows the simulated and measured results of the FRAA normalized radiation pattern under uniform feeding conditions. The sidelobe level simulation results of FRAA operating in X-band, $\mathrm{Ku}$-band, and $\mathrm{Ka}$-band are $-15.1 \mathrm{~dB}$, $-14.9 \mathrm{~dB}$, and $-14.3 \mathrm{~dB}$, respectively. The results of the 
TABLE 2: Comparison of the FRAA with other multiband/switchable-band antenna arrays.

\begin{tabular}{|c|c|c|c|c|c|}
\hline Item & Our work & Ref. [14] & Ref. [16] & Ref. [19] & Ref. [18] \\
\hline Reconstruction band & $\mathrm{X} / \mathrm{Ku} / \mathrm{Ka}$ & $\mathrm{X} / \mathrm{Ku} / \mathrm{Ka}$ & $\mathrm{L} / \mathrm{S}$ & $\mathrm{L} / \mathrm{C}$ & $\mathrm{X} / \mathrm{Ku}$ (multiband) \\
\hline Frequency ratio & $3.57: 1$ & $3.12: 1$ & $2.2: 1$ & $3.2: 1$ & $1.8: 1$ \\
\hline Number of element switches & 2 & 4 & 4 & 16 & 0 \\
\hline Scanning range & $20^{\circ}$ & $0^{\circ}$ & $\pm 60^{\circ}$ & $\pm 60^{\circ}$ & $50^{\circ}$ \\
\hline Is the amplitude weighted & No & No & No & Not mentioned & No \\
\hline Sidelobe level simulation result $(\mathrm{dB})$ & $-15.7 @ 20^{\circ}$ & Not mentioned & Around-12.5@15 & Around -12.0@0 & -14.0 (synthetic) \\
\hline Element size $\left(\lambda_{\mathrm{L}}\right)$ & $0.14 \times 0.35$ & $0.14 \times 0.13$ & $0.22 \times 0.22$ & $0.18 \times 0.18$ & $0.33 \times 0.33$ \\
\hline Element spacing $\left(\lambda_{\mathrm{L}} / \lambda_{\mathrm{H}}\right)$ & Irregular & $0.4 / 1.27$ & $0.5 / 0.5$ & $0.36 /<0.5$ & $0.33 / 0.58$ \\
\hline
\end{tabular}

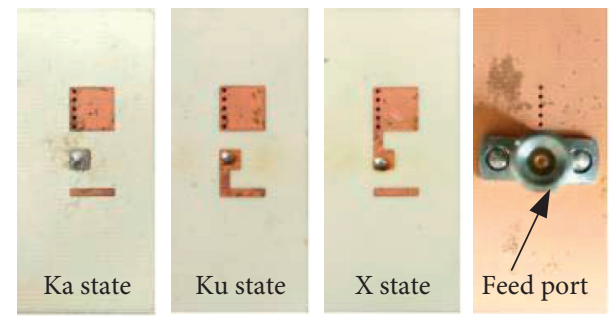

FIGURE 16: The fabricated FRA prototypes.

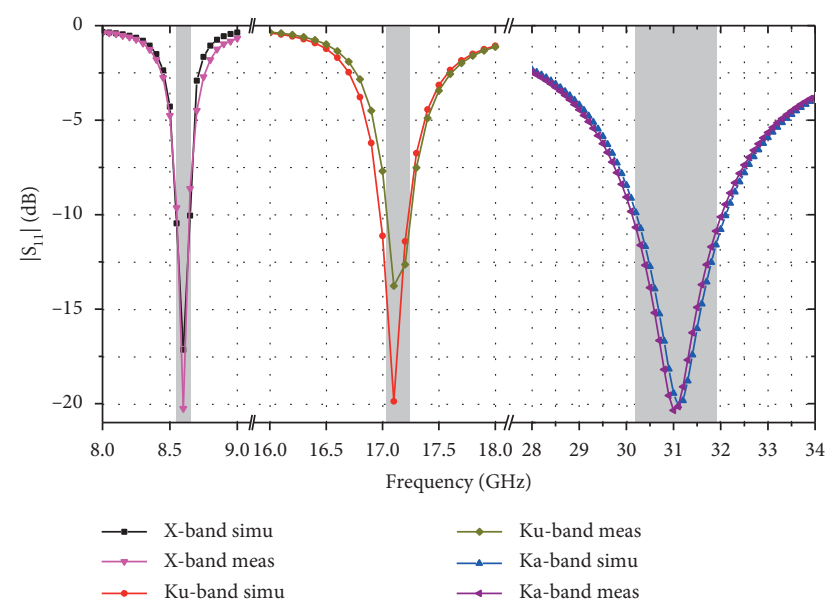

FIgURE 17: Measured reflection coefficients of FRA prototype working at different states.

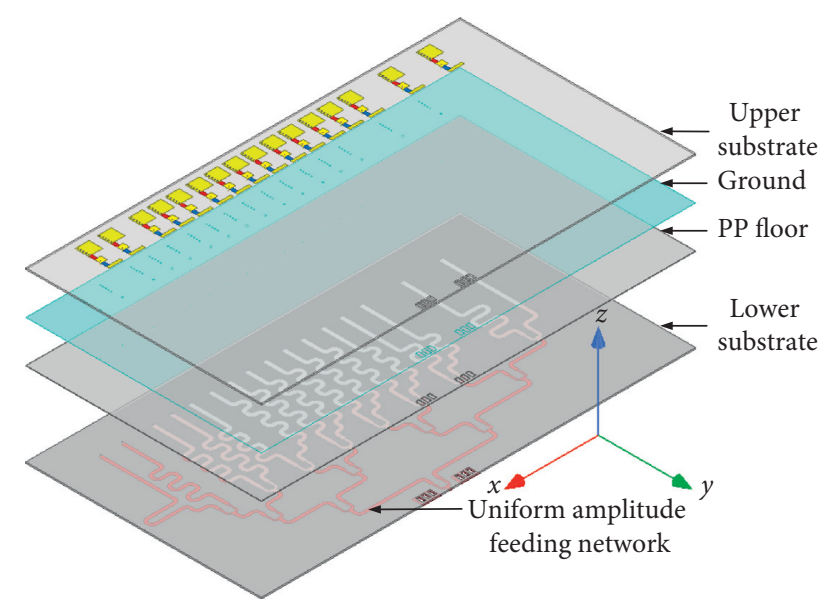

FIGURE 18: Structure of the FRAA prototype with uniform amplitude feeding network.

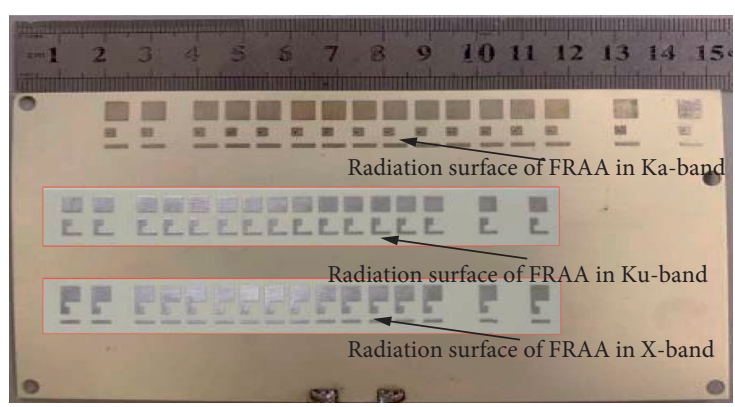

(a)

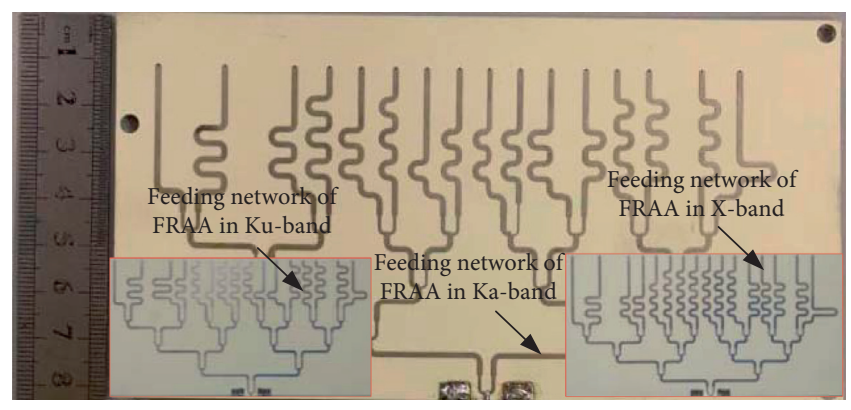

(b)

FIgURE 19: The fabricated $1 \times 16$ linear irregular FRAA prototype. (a) Radiation surface. (b) Feeding network surface.

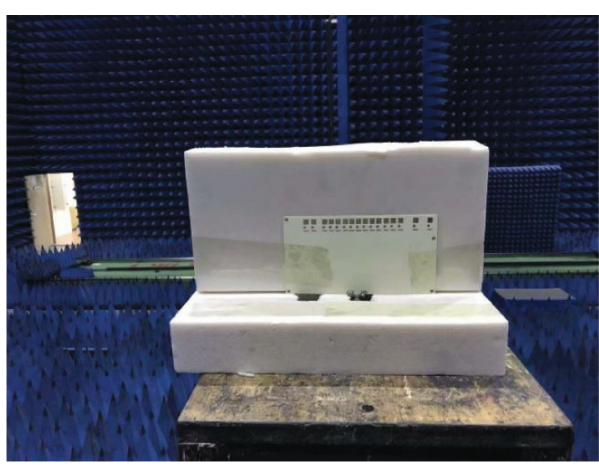

FIGURE 20: The measured scene.

sidelobe level measurements are $-13.6 \mathrm{~dB},-13.1 \mathrm{~dB}$, and $-12.1 \mathrm{~dB}$, respectively. Due to the testing environment error, the sidelobe level is slightly increased, which shows that the sidelobe level increases with the increase of the operating frequency. On the whole, the simulated and measured results are in good agreement. 


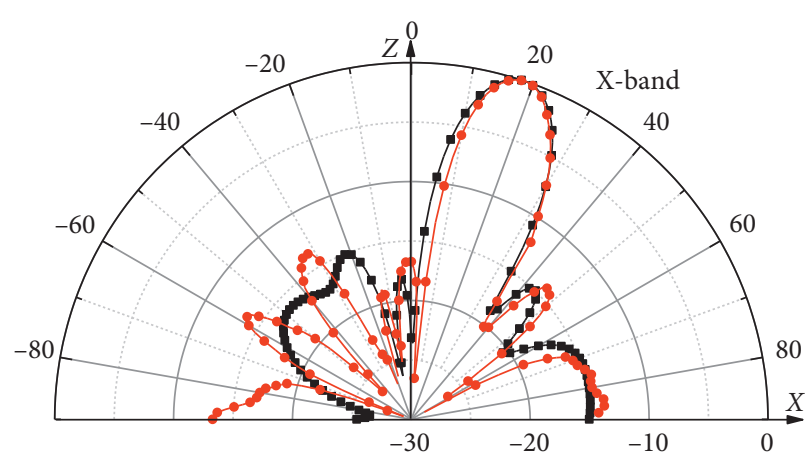

$(\mathrm{dB})$

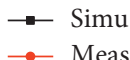

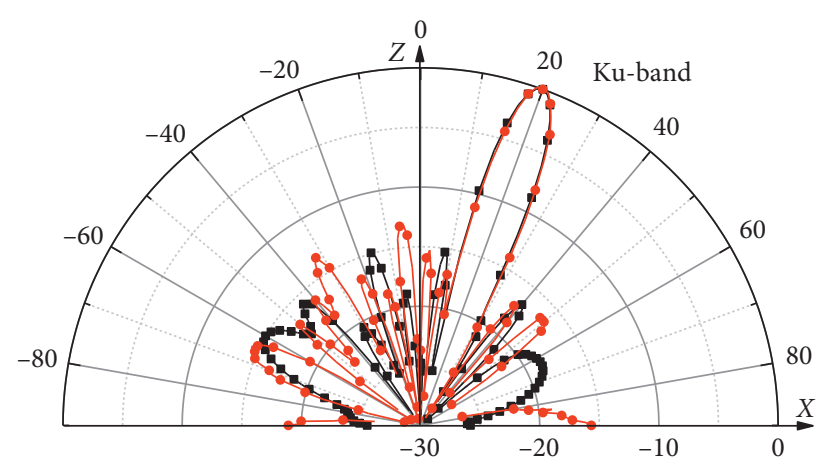

$(\mathrm{dB})$

(a)

(b)

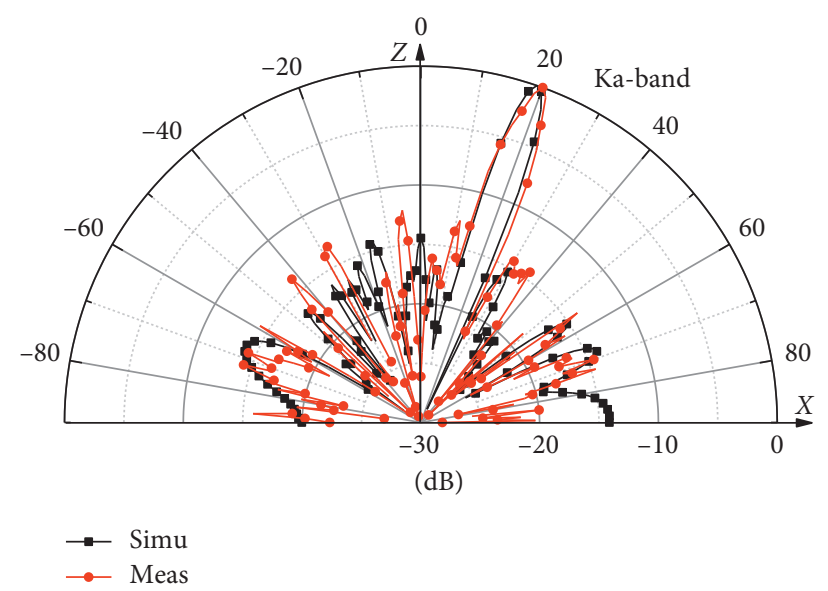

(c)

FIGURE 21: The normalized radiation pattern measured and simulated results of FRAA prototypes in different frequency reconstruction states. (a) X-band. (b) Ku-band. (c) Ka-band.

\section{Conclusion}

In this paper, a frequency reconfigurable antenna element integrating two RF MEMS switches has been proposed. The FRA element has the reconstruction capability in X-band, $\mathrm{Ku}-$ band, and Ka-band, and the frequency reconstruction ratio reaches $3.57: 1$. Besides, the miniaturization design of the element has been achieved through five metal shorting holes between the radiating surface and the ground plate, reaching $0.14 \lambda_{\mathrm{X}} \times 0.35 \lambda_{\mathrm{X}}$, providing a good structural basis for the frequency reconfigurable antenna array. By employing intelligent algorithm and MATLAB-HFSS joint optimization, a $1 \times 16$ linear irregular FRAA based on the FRA element has been proposed, which realizes that beam deflection in the same direction corresponds to different frequency reconstruction state. In addition, compared with the traditional uniformly spaced FRAA, the sidelobe level of the irregular antenna line array is improved by not less than $3 \mathrm{~dB}$, and the grating lobe in the Ka band is effectively avoided. Finally, both FRA element and FRAA prototypes have been fabricated and tested to verify their superiority. The measurement results show that the proposed trifrequency reconfigurable linear irregular FRAA has broad application prospects in the future multifrequency wireless communication and intelligent radar anti-interference fields.

\section{Data Availability}

The data used to support the findings of this study are included within the article.

\section{Conflicts of Interest}

The authors declare that there are no conflicts of interest regarding the publication of this paper.

\section{Acknowledgments}

This work was supported by the National Natural Science Foundation of China under Grant 61774096.

\section{References}

[1] IEEE, "IEEE standard definitions of terms for antennas," IEEE Std, vol. 145-2013, 2013. 
[2] L. Ge and K.-M. Luk, "A band-reconfigurable antenna based on directed dipole," IEEE Transactions on Antennas and Propagation, vol. 62, no. 1, pp. 64-71, 2014.

[3] C. R. White and G. M. Rebeiz, "A shallow varactor-tuned cavity-backed slot antenna with a 1.9:1 tuning range," IEEE Transactions on Antennas and Propagation, vol. 58, no. 3, pp. 633-639, 2010.

[4] C.-Y. Chiu, J. Li, S. Song, and R. D. Murch, "Frequencyreconfigurable pixel slot antenna," IEEE Transactions on Antennas and Propagation, vol. 60, no. 10, pp. 4921-4924, 2012.

[5] M. R. Hamid, P. Gardner, P. S. Hall, and F. Ghanem, "Switched-band vivaldi antenna," IEEE Transactions on Antennas and Propagation, vol. 59, no. 5, pp. 1472-1480, 2011.

[6] A. Boukarkar, X. Q. Lin, Y. Jiang, and X. F. Yang, "A compact frequency-reconfigurable 36-states patch antenna for wireless applications," IEEE Antennas and Wireless Propagation Letters, vol. 17, no. 7, pp. 1349-1353, 2018.

[7] C. Sun, H. Zheng, L. Zhang, and Y. Liu, "A compact frequency reconfigurable patch antenna for Beidou (COMPASS) navigation system," IEEE Antennas Wireless Propagation Letters, vol. 13, pp. 967-970, 2014.

[8] Y. K. Choukiker and S. K. Behera, "Wideband frequency reconfigurable Koch snowflake fractal antenna," IET Microwaves, Antennas \& Propagation, vol. 11, no. 2, pp. 203-208, 2017.

[9] A. Grau, M.-J. Lee, J. Romeu, H. Jafarkhani, L. Jofre, and F. De Flaviis, "A multifunctional mems-reconfigurable pixel antenna for narrowband mimo communications," in Proceedings of the 2007 IEEE Antennas and Propagation Society International Symposium, Honolulu, HI, USA, June 2007.

[10] D. Rodrigo, Y. Damgaci, N. Biyikli, B. A. Cetiner, J. Romeu, and L. Jofre, "MEMS-reconfigurable antenna based on a multi-size pixelled geometry," in Proceedings of the Fourth European Conference on Antennas and Propagation (EuCAP), IEEE, Barcelona, Spain, pp. 1-4, April 2010.

[11] M. D. Wright, W. Baron, J. Miller, J. Tuss, D. Zeppettella, and M. Ali, "MEMS reconfigurable broadband patch antenna for conformal applications," IEEE Transactions on Antennas and Propagation, vol. 66, no. 6, pp. 2770-2778, 2018.

[12] H. Yang, F. Yang, X. Cao et al., "A 1600-element dual-frequency electronically reconfigurable reflectarray at $\mathrm{X} / \mathrm{Ku}-$ band," IEEE Trans. Antennas Propag.vol. 65, no. 6, pp. 3024-3032, 2017.

[13] R. L. Jordan, B. L. Huneycutt, and M. Werner, "The SIR-C/XSAR synthetic aperture radar system," IEEE Transactions on Geoscience and Remote Sensing, vol. 33, no. 4, pp. 829-839, 1995.

[14] L. Li, Z. Wu, K. Li et al., "Frequency-reconfigurable QuasiSierpinski antenna integrating with dual-band high-impedance surface," IEEE Transactions on Antennas and Propagation, vol. 62, no. 9, pp. 4459-4467, 2014.

[15] J. A. Byford, K. Y. Park, P. Chahal, and E. J. Rothwell, "Frequency reconfigurable patch antenna array," Electronics Letters, vol. 51, no. 21, pp. 1628-1630, 2015.

[16] N. Haider, A. G. Yarovoy, and A. G. Roederer, “\$L/S\$ -band frequency reconfigurable multiscale phased array antenna with wide angle scanning," IEEE Transactions on Antennas and Propagation, vol. 65, no. 9, pp. 4519-4528, 2017.

[17] Q. Li, T. Li, Z. Li, and J. Fang, "Unidirectional frequency reconfigurable bow-tie antenna array with AMC reflector," in Proceedings of the 11th European Conference on Antennas and Propagation (EUCAP), pp. 2221-2223, Paris, France, March 2017.
[18] S. E. Valavan, D. Tran, A. G. Yarovoy, and A. G. Roederer, "Dual-band wide-angle scaning planar phased array in $\mathrm{X} / \mathrm{Ku}-$ bands," IEEE Transactions on Antennas and Propagation, vol. 62, no. 5, pp. 2514-2521, 2014.

[19] M. Shirazi, J. Huang, T. Li, and X. Gong, "A switchablefrequency slot-ring antenna element for designing a reconfigurable array," IEEE Antennas and Wireless Propagation Letters, vol. 17, no. 2, pp. 229-233, 2018.

[20] K.-L. Wong, Compact and Broadband Microstrip Antenna, John Wiley \& Sons, New York, NY, USA, 2002.

[21] M. Li, J. Zhao, Z. You, and G. Zhao, "Design and fabrication of a low insertion loss capacitive RF MEMS switch with novel micro-structures for actuation," Solid-State Electronics, vol. 127, pp. 32-37, 2017.

[22] D. Parker and D. C. Zimmermann, "Phased arrays-part 1: theory and architectures," IEEE Transactions on Microwave Theory and Techniques, vol. 50, no. 3, pp. 678-687, 2002.

[23] X. Yuan, Z. Li, D. Rodrigo et al., "A parasitic layer-based reconfigurable antenna design by multi-objective optimization," IEEE Transactions on Antennas and Propagation, vol. 60, no. 6, pp. 2690-2701, 2012.

[24] A. Darvish and A. Ebrahimzadeh, "Improved fruit-fly optimization algorithm and its applications in antenna arrays synthesis," IEEE Transactions on Antennas and Propagation, vol. 66, no. 4, pp. 1756-1766, 2018. 